\title{
Larval Geoduck (Panopea generosa) Proteomic Response to Ciliates
}

\author{
Emma Timmins-Schiffman ${ }^{1}$, José M. Guzmán ${ }^{2}$, Rhonda Elliott Thompson ${ }^{3,4}$, \\ Brent Vadopalas $^{2}$, Benoit Eudeline ${ }^{3} \&$ Steven B. Roberts ${ }^{2 *}$
}

The innate immune response is active in invertebrate larvae from early development. Induction of immune response pathways may occur as part of the natural progression of larval development, but an up-regulation of pathways can also occur in response to a pathogen. Here, we took advantage of a protozoan ciliate infestation of a larval geoduck clam culture in a commercial hatchery to investigate the molecular underpinnings of the innate immune response of the larvae to the pathogen. Larval proteomes were analyzed on days 4-10 post-fertilization; ciliates were present on days 8 and 10 post-fertilization. Through comparisons with larval cultures that did not encounter ciliates, proteins implicated in the response to ciliate presence were identified using mass spectrometry-based proteomics. Ciliate response proteins included many associated with ribosomal synthesis and protein translation, suggesting the importance of protein synthesis during the larval immune response. There was also an increased abundance of proteins typically associated with the stress and immune responses during ciliate exposure, such as heat shock proteins, glutathione metabolism, and the reactive oxygen species response. These findings provide a basic understanding of the bivalve molecular response to a mortality-inducing ciliate and improved characterization of the ontogenetic development of the innate immune response.

Bivalve invertebrates possess an innate immune response that can be triggered by biotic threats, including pathogens. There are three steps in the innate immune response including immune recognition, immune signaling, and immune effectors ${ }^{1}$. Once the response is activated, proteins are upregulated that are essential in immune defense and pathogen removal. Adult bivalves are able to activate the entire suite of proteins necessary for a successful immune response, but larvae may have less developed response that makes them more vulnerable to infection and mortality ${ }^{2}$. Increased vulnerability has been observed in differential mortality rates between developmental stages of the same bivalve species upon exposure to the same pathogen ${ }^{3,4}$.

The larval bivalve immune system may begin developing in the embryo within hours post-fertilization ${ }^{2}$. Gene transcripts and proteins involved in the larval immune response are expressed throughout developmental stages, and likely play important roles in larval development as dramatic tissue restructuring occurs ${ }^{2,5-10}$.

Ciliates are widespread in aquatic systems and are known harmless parasites of many species of bivalves, but some species are capable of causing significant mortality. Ciliate parasites have been observed in multiple species of bivalves in hatchery and in wild settings ${ }^{11-13}$. In zebra mussels, increased ciliate infestations were associated with moribund/dead mussels, but the authors hypothesized that the ciliates opportunistically feed on dead and dying tissue from a distinct, primary infection ${ }^{11}$. Ciliate outbreaks in hatcheries can also occur when bacterial communities are unchecked by antibiotics ${ }^{7}$. Bacterivorous ciliates can impact the settlement rates of invertebrate larvae through secondary impacts on water currents above the settlement surface or selective grazing of specific bacteria $^{14}$. Ciliates are known to be more prevalent in hatcheries ${ }^{12}$ where they enter in bulk water and persist in the system ${ }^{15}$.

The geoduck clam (Panopea generosa) is a burrowing hiatellid clam found in low intertidal and subtidal sediments throughout the Northeast Pacific coast, including the US (Alaska, Washington, California), Canada (British Columbia), and Mexico (North Baja Pacific Coast) ${ }^{16-18}$. In addition to their economic importance, geoduck are abundant and essential environmental engineers in their natural habitat ${ }^{19,20}$. Geoduck larvae and adults are susceptible to parasites in the Puget Sound, WA region ${ }^{21,22}$. Having established a baseline proteome for

\footnotetext{
${ }^{1}$ University of Washington, Department of Genome Sciences, 3720 15th Ave NE, Seattle, WA, 98195, United States. 'University of Washington, School of Aquatic and Fishery Sciences, 1122 Boat St., Seattle, WA, 98195, United States. ${ }^{3}$ Taylor Shellfish Hatchery, 701 Broadspit Rd., Quilcene, WA, 98376, United States. ${ }^{M}$ Mason County Public Health, 415N 6th St., Shelton, WA, 98584, United States. *email: sr320@uw.edu
} 


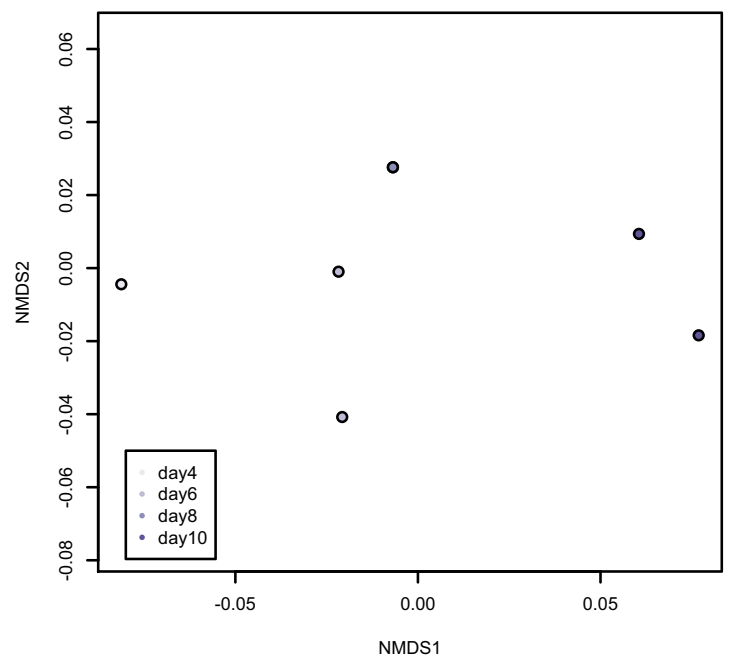

Figure 1. Nonmetric multidimensional scaling plot of larval proteomes $(\mathrm{N}=6,133$ proteins) across days 4 through 10 post-fertilization. The points are lightest on day 4 and darkest on day 10 . The two points representing proteomes on day 8 are directly on top of each other.

geoduck larval development in previous work ${ }^{23}$, we sought to better characterize the geoduck immune response to ciliate pathogens.

In this study, we take advantage of an opportunistic infestation of a putative parasitic ciliate in a commercial hatchery to characterize for the first time the immune response of geoduck clam larvae during veliger development (days 4-10 post-fertilization). This was accomplished using liquid chromatography coupled with high-resolution tandem mass spectrometry that allowed us to build a proteomic profile of larval development and characterize the induced innate immune response in the veliger larvae.

\section{Results}

Larval growth and mortality. By day 4 post-fertilization, geoduck larvae had an average shell size of $131 \mu \mathrm{m}$. This increased to $146 \mu \mathrm{m}$ on day 6 and $161 \mu \mathrm{m}$ on day 8 post-fertilization. At day 4 post-fertilization, 3.1 million larvae were stocked in each tank. By day 10 post-fertilization there were 516,000 larvae in one tank and 1.3 million in the other. Ciliates were observed in the larval samples by day 8 post-fertilization; by day 10 , there were high numbers of ciliates in the larval culture accompanied by high larval mortality.

Proteomics of larval development. The abundance of proteins in the geoduck larval proteomes $(\mathrm{N}=6,133$ proteins; Supplementary Table S1) are significantly distinct from each other across days 4 through 10 post-fertilization (ANOSIM R $=0.8889$, p-value $=0.005$; Fig. 1). Four proteins are strongly, positively weighted on NMDS1 axis and thus contribute significantly to proteomic changes over time - elongation factor 1 a (geoduck protein ID in Supplementary Table S1 DN61152_c1_g2_i1::g.31740), deleted in malignant brain tumors 1 protein (DN55033_c0_g1_i1::g.9300), peptidyl-prolyl cis-trans isomerase (DN40375_c0_g1_i1::g.39279), and tubulin alpha chain (DN61033_c0_g2_i3::g.6327).

The strongest signal in the differentially abundant enrichment analysis comes from the changing abundance of proteins involved in translation over time. Many of these proteins increase in abundance over time. Between days 6 and 8 post-fertilization, proteins at elevated abundance on day $8(n=38$ proteins) contribute to the enrichment of GO terms translational elongation (biological process, $\mathrm{BP}$ ), and translational elongation factor activity (molecular function, MF). Between days 8 and 10, proteins at relatively higher abundance on day $10(\mathrm{n}=138$ proteins) are responsible for the enriched terms translation (BP), translational elongation (BP), ribosome (cellular component, CC), cytosolic large ribosomal subunit (CC), small ribosomal subunit (CC), structural constituent of ribosome (MF), and rRNA binding (MF).

Proteomic profile of larval development. Proteins regulating the progress of larval development were identified through comparison with similar proteomes from larvae raised at lower $\mathrm{pH}$ that did not experience ciliate-induced mortality. Functional categories identified in both datasets were determined to be involved in development, as opposed to the stress response. Diverse protein groups made up the full suite of differentially abundant proteins across developmental time. Proteins at elevated abundance on day 6 compared to day $8(\mathrm{n}=28$ proteins) included those involved in muscle activity, cytoskeletal structure, amino acid degradation, and carbohydrate metabolism. Proteins involved in muscle activity were four isoforms of twitchin (DN4488 c0_g1_i1::g.37991, DN84882_c0_g1_i1::g.7474, DN37585_c0_g1_i1::g.30792, DN54513_c0_g1_i1::g.20073). Cytoskeletal structure proteins included an axonemal $84 \mathrm{kDa}$ protein (DN52327_c0_g1_i1::g.16221), two isoforms of spectrin beta chain (DN49196_c0_g1_i1::g.12832, DN63615_c0_g1_i4::g.19798), and dystonin (DN64009_c1_g1_i4::g.9078). An enzyme involved in glycine degradation (glycine cleavage system P protein DN58628_c5_g1_i2::g.33280) and lysine degradation (alpha-aminoadipic semialdehyde synthase - DN102693_ c0_g1_i1::g.40254) were elevated on day 6. The up-regulation of carbohydrate metabolism was supported by the 
inclusion of malic enzyme (DN55577_c7_g1_i1::g.30103), endoglucanase (DN59810_c3_g1_i1::g.27515), and transketolase (DN62420_c0_g1_i1::g.26365).

On day 8, proteins at increased abundance were involved in processes such as embryonic development, translation, stress response, and the cell cycle. Mesenchyme-specific cell surface glycoprotein (compared to day 6, DN60034_c1_g1_i1::g.37906), agrin (compared to days 6 and 10, DN50844_c0_g1_i1::g.3048), and delta-like protein (compared to day 10, DN64019_c1_g1_i2::g.9096) were at elevated abundance on day 8 and are responsible for tissue development and neuromuscular junctions during embryogenesis. Several proteins involved in protein translation, transport, and degradation were at higher levels on day 8, including ribosomal proteins (e.g., DN55459_c0_g2_i2::g.4318), elongation factors (e.g., DN61142_c1_g2_i1::g.31740), nucleolar protein 56 (DN57041_c0_g2_i1::g.3336), RNA polymerase-II associated factor (DN57191_c0_g1_i2::g.28950), and cathepsins (e.g., DN54782_c0_g1_i1::g.5290). On day 8 there may have been an up-regulation of the cell cycle with evidence from increased abundance (compared to day 10) of proteins involved in cell cycle control and the cytoskeleton (e.g., centrosomal protein of $78 \mathrm{kDa}-$ DN44281_c0_g1_i2::g.1342, nuclear protein MDM1 DN64001_c0_g1_i1::g.14401, antigen KI-67 - DN63195_c0_g2_i2::g.25524, suppressor of G2 allele of SKP1-like protein - DN63050_c5_g2_i1::g.279). Apoptosis proteins were at elevated abundance, including cell division cycle and apoptosis regulator protein 1 (compared to day 10, DN55421_C0_g1_i1::g.4308) and programmed cell death protein 4 (compared to days 6 and 10, DN60246_c2_g3_i2::g.7173). Cilia biogenesis was also important at this time point with an increased abundance of proteins such as centrin-4 (DN30560_c0_g1_i2::g.39913) and dynein heavy chain 7, axonemal (DN52275_c0_g1_i1::g.16099), cilia- and flagella-associated protein 58 (DN63766_c0_ g1_i4::g.4722), and intraflagellar transport protein 57-like (DN62855_c0_g2_i1::g.20768). Four of the proteins that were at elevated abundance on day 8 were glycoproteins: mesenchyme-specific cell surface glycoprotein (DN60034_c1_g1_i1::g.37906), prosaposin (DN63880_c2_g1_i3::g.30762), agrin (DN50844_c0_g1_i1::g.3048), and fibronectin type-III domain-containing protein (DN55720_c0_g1_i1::g.14093).

The most significant proteomic signal on day 10 was a surge in abundance of ribosomal proteins with other proteins at higher abundance in categories such as protein folding, tissue growth/restructuring, and energy metabolism. Dozens of ribosomal proteins, several elongation factors, and other proteins associated with translation were at increased abundance on day 10. As a likely balance to the surge of new proteins, several proteins involved in protein degradation (aminopeptidase N - DN60972_c0_g2_i1::g.12028, cysteine peptidase - DN24514_c0_g1_i1::g.3095) and protein folding (a suite of heat shock proteins - e.g., DN56353_ c0_g1_i1::g.29541, peptidyl-prolyl cis-trans isomerase - DN40375_c0_g1_i1::g.39279, calreticulin - DN42620_ c0_g1_i1::g.2821, and glucose-regulated protein 78 - DN41738_c0_g1_i2::g.3144) were also detected at elevated levels on day 10. There is also evidence of tissue restructuring with an increase in abundance of proteins involved in muscle growth (kyphoscoliosis peptidase - DN54342_c0_g1_i1::g.35621, actin - DN40952_c0_g1_i1::g.3693, twitchin - DN84882_c0_g1_i1::g.7474), vascularization (basement membrane-specific heparan sulfate proteoglycan core protein - DN1574_c0_g1_i1::g.30025), and embryogenesis (multiple epidermal growth factor-like domains protein - DN61224_c0_g1_i1::g31991). There was also an increase in abundance of proteins involved in metabolism and energy production: acetyl-CoA C-acyltransferase, succinate-CoA ligase [ADP/GDP-forming] subunit alpha, ATP/ADP translocase, and adenosine kinase (DN65641_c0_g1_i1::g.38953, DN5111_c0_g1_ i1::g.3560, DN31265_c0_g1_i1::g.39364, DN86572_c0_g1_i1::g.31553).

Proteomic profile of larval immune response. There were 855 proteins identified in the $\mathrm{pH} 8.2$ dataset that were not detected in larvae reared in parallel at lower $\mathrm{pH}$ where there was no ciliate presence. Some of these proteins would have been detected in the current dataset because of their role in ciliate response; others may have been unique to this dataset simply due to the difference in ambient $\mathrm{pH}$ of the larval culture. In the enrichment analysis, compared to all proteins in the $\mathrm{pH} 8.2$ dataset, enriched terms included integral component of membrane (CC), membrane (CC), regulation of transcription DNA-templated (BP), and DNA-binding transcription factor activity (MF). Eleven of these proteins were significantly differentially abundant (Fig. 2), 10 of which were highest at day 10 and most of which are ribosomal proteins.

On day 8 , ciliates were first observed in the larval culture. In addition to the proteins described in the previous section, stress response proteins also increased on day 8 compared to days 6 and 10, including two isoforms of heat shock protein 70 (DN56353_c0_g1_i1::g.29541, DN56353_c0_g1_i2::g.29542), a thaumatin-like protein (DN55757_c0_g1_i1::g.14062), glutamate-cysteine ligase catalytic subunit (DN62819_c0_g1_i1::g.20739), glutathione peroxidase (DN60231_c1_g1_i1::g.7155), glutathione reductase (DN61098_c8_g3_i1::g.6407), and superoxide dismutase (DN61210_c0_g1_i3::g.32050) (Fig. 2).

The ciliate infestation had increased by day 10, along with larval mortality (see above). The parasite virulence factor cruzipain (DN76433_c0_g1_i1::g.22190) was first detected on day 8 in the proteomics data and was at significantly elevated abundance by day 10 (Fig. 2).

\section{Discussion}

Geoduck larvae have distinct proteomic profiles based on developmental stage with evidence of a molecular response to protozoan ciliate infestation. Most of the differentially abundant proteins between developmental time points were likely involved in the progression of larval development since they represented similar functional groups to those proteins detected in parallel larval cultures. Eleven larval proteins and one protozoan protein emerged as likely markers of ciliate infestation of larval cultures. Over 800 proteins were detected in the current dataset of larvae reared at $\mathrm{pH} 8.2$ and were not detected at lower $\mathrm{pH}$, which included many proteins involved in protein translation; those that were significantly differentially abundant increased in abundance during the ciliate infestation (days 8 through 10 post-fertilization). This dataset further establishes the timeline of larval molecular development and also elucidates some of the mechanisms deployed by the larval immune response. 
A

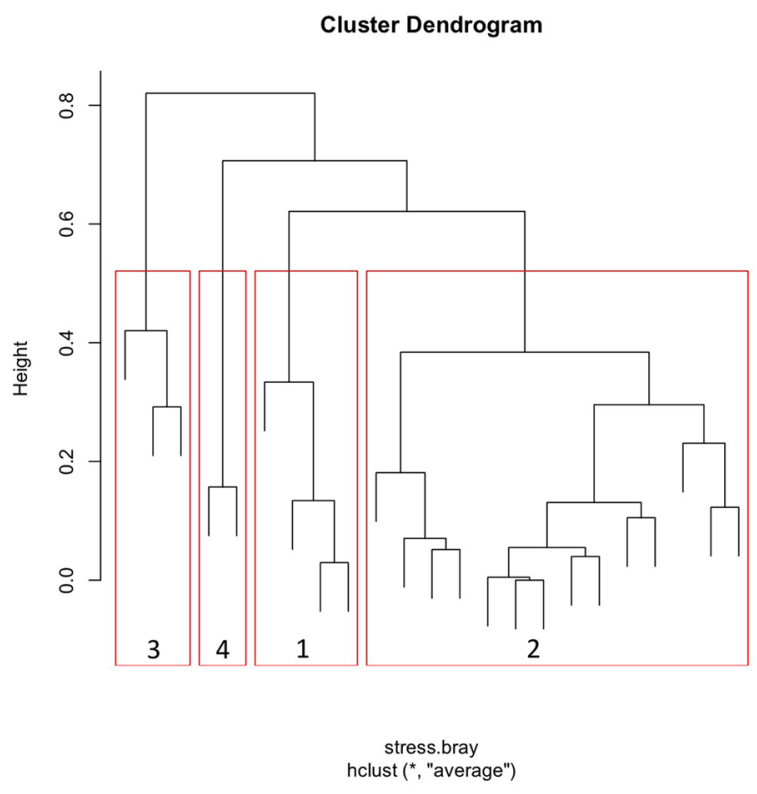

B

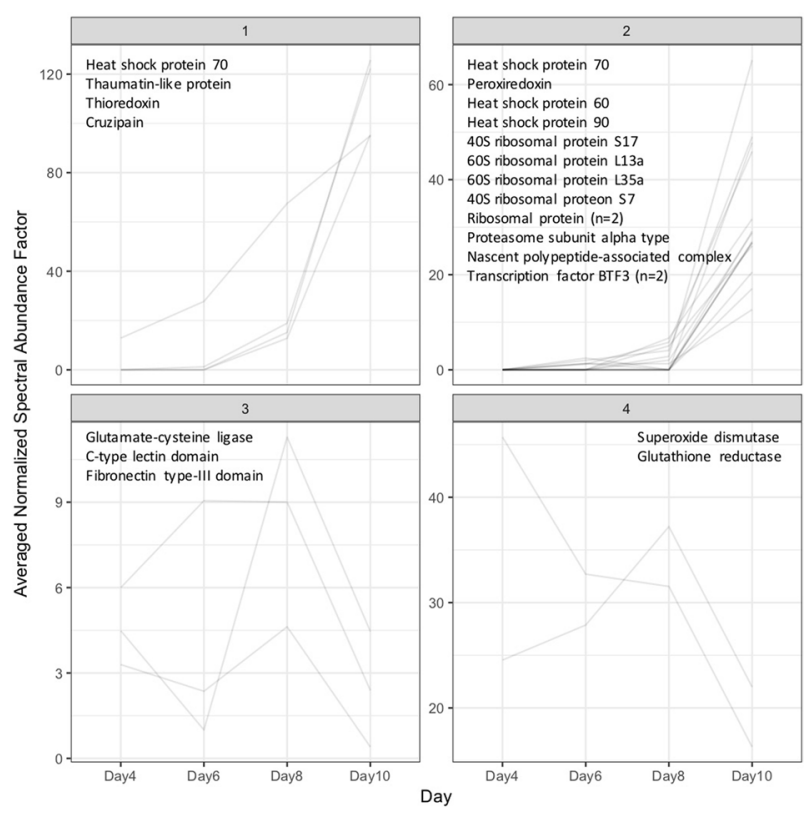

Figure 2. Proteins hypothesized to be involved in the larval stress response to ciliate infestation. The clustered protein group dendrogram (panel A) shows the 4 clusters of proteins in red boxes with the cluster number at the bottom of each box. Panel B shows the abundance profile for each protein across day 4-10 post-fertilization with abundance plots grouped by cluster (cluster number is in the header of each plot). Annotations for the proteins in each plot are listed within each plot. All proteins are likely geoduck larval proteins except for cruzipain (cluster 1), which is a parasitic virulence protein.

Protein abundance patterns across time points in larvae reared at $\mathrm{pH} 8.2$ generally followed the established timeline for geoduck at $\mathrm{pH}$ 7.5. Even though we compared larvae across different $\mathrm{pH}$, the larvae reared at $\mathrm{pH} 7.5$ are an appropriate direct comparison for this study because 1) baseline proteomic trends of development were maintained across the two $\mathrm{pH}, 2$ ) they are from the same spawning event, and 3) the larvae at both $\mathrm{pH}$ developed normally (there was no sign of adverse impacts of $\mathrm{pH}$ on morphology at $\mathrm{pH}$ 7.5). Changes in abundance of proteins involved in protein metabolism and translation dominated the results of proteomic differences across larval development. This trend was apparent in the analysis of the NMDS (Fig. 1), where two of the proteins strongly weighted along axis 1were associated with translation (elongation factor 1a) and protein folding (peptidyl-prolyl cis-trans isomerase). Additionally, many of the differentially abundant proteins between time points were associated with translation and protein metabolism, a trend also observed in geoduck larvae reared at lower $\mathrm{pH}^{23}$. The maintenance and upregulation of translation, even under the stress of ciliate infestation, underlines the physiological drive of the developmental processes that rely on new proteins to grow and create new tissue structures, but likely also underpins the immune/stress response to ciliates (see below). At day 6 post-fertilization there was increased abundance of proteins involved in muscle activity, cytoskeletal structure, amino acid degradation, and carbohydrate metabolism. At day 8 post-fertilization, proteins involved in embryonic development, translation, and the cell cycle increased. Increased functional categories at day 10 post-fertilization included translation, along with increased abundances of proteins involved in tissue growth and energy metabolism. A full discussion of the significance of these changes can be found in [23].

Ciliates were detected in the larval culture at day 8 post-fertilization and starting at this time point there was molecular evidence of a larval stress and immune response. Some of these stress response proteins peaked in abundance at day 8 and some at day 10 (Fig. 2), at which point the ciliate infestation was more intense and significant larval mortality was observed. The hypothesized larval proteomic response to ciliates included an increase in abundance of the molecular chaperones heat shock proteins and of proteins involved in the reactive oxygen species (ROS) response. Immune response proteins are known to be active during larval development and metamorphosis $^{5,24}$. Several proteins annotated with GO terms "immune response" and "innate immune response" were detected at pH 8.2 and in the pH 7.5 dataset: interleukin enhancer-binding factor-2 like (DN57893_c2_g2_ i1::g.33736), tubulointerstitial nephritis antigen-like (DN61593_c0_g3_i1::g.26870), RPE-spondin (DN63587_ c1_g2_i1::g.19583), peptidoglycan-recognition protein (DN54849_c0_g1_i1::g.30972), and protein toll (DN101391_c0_g1_i1::g.40331). The primary role of immune response proteins in development is hypothesized to be recognizing and responding to dying tissue during tissue resorption and organogenesis, but many of these proteins are also induced upon pathogen exposure ${ }^{2,5-9}$. Embryonic immunity is derived from maternal peptides and proteins $s^{2,10}$, but, starting at the gastrula and trochophore stages, bivalve larvae produce their own immune response proteins with immune competence reached between the $\mathrm{D}$-hinge and veliger stages ${ }^{2}$.

Heat shock proteins (HSPs) are significant participants in the invertebrate immune response and increased levels of these proteins were detected on days 8 and 10 post-fertilization in this study, with highest abundances 
at day 10. The suite of differentially abundant heat shock proteins included two isoforms of HSP70, chaperoning HSP60, and HSP90. In marine fish and invertebrates, HSP upregulation occurs in response to any external stressor, including disease ${ }^{25}$, likely as a response to stress-induced tissue and protein damage. These molecular chaperones may play roles in intercellular signaling as well as chaperoning peptides released from dead and dying tissues $^{25}$. This conserved response is activated across bivalve larvae. In veliger Pacific oysters gene expression of hsp70 increased upon infection by the bacteria Vibrio corallilyticus ${ }^{9}$. In oyster larvae untreated with antibiotics, gene expression levels of $h s p 70$ were elevated a week before a mass mortality event, but decreased immediately before larval mortality occurred ${ }^{8}$. In the current study, geoduck larvae were not fighting off a known bacterial infection, but were responding to attack from ciliates. It may be that an upregulation of heat shock proteins was part of a general immune response upregulation, or a response to the tissue damage incurred by ciliates.

Reactive oxygen species (ROS) are a well-established component of the invertebrate immune response and response to these damaging molecules can be seen in the proteomic profiles of the larvae. Thioredoxin, peroxiredoxin-1, and superoxide dismutase were all detected at elevated abundances on day 8 and/or 10 post-fertilization. These proteins are well-known for their roles in responding to ROS to reduce tissue damage. Additionally, glutamate-cysteine ligase, glutathione reductase and glutathione peroxidase, all part of the glutathione metabolism pathway, were increased on day 8 post-fertilization, compared to day 10. Glutathione is an essential part of the cellular response to ROS to prevent cellular damage ${ }^{26}$. Adult bivalves are known to up-regulate ROS production as a defense mechanism against pathogens ${ }^{27}$ and there is evidence that bivalve larvae do the same as early as the trochophore stage ${ }^{6,7}$. When molluscan hemocytes detect a pathogen, they undergo respiratory burst, which generates free radicals such as $\mathrm{ROS}^{28}$. In oyster larvae, gene expression and enzymatic activity of superoxide dismutase, catalase (another ROS defense protein), peroxiredoxin, and the glutathione pathway were all elevated in response to pathogen exposure in veligers ${ }^{7-9}$. Geoduck larvae seem to effect the same molecular cascade of hemocyte respiratory burst, followed by ROS mitigation, in their response to ciliates.

An apparent upregulation of protein translation during the ciliate infestation was evidenced by the differential abundance of several of these proteins during exposure to ciliates and detected only in larvae reared at pH 8.2. Protein translation underlies any type of proteomic upregulation as new proteins are synthesized in response to a stimulus. Protein translation in the eukaryotic host is an essential part of the immune and stress responses ${ }^{29}$. It may be that these differentially abundant proteins ( 6 ribosomal proteins, proteasome subunit alpha type, nascent polypeptide-associated complex, and 2 transcription factors BTF3) were detected at increased abundance coincident with ciliate presence because they are the foundation of immune physiology upregulation as larvae attempt to defend themselves against the parasite. They may also be evidence of the unfolded protein response and endoplasmic reticulum stress ${ }^{30}$, although many of the molecular hallmarks of this response were not detected in the differentially abundant proteins. It is clear that the larval immune response encompasses a network of proteins that work together to launch a physiological change and that this network extends beyond the typical biomarkers selected for single protein or enzyme assays.

The proteomic signals of a physiological stress response and coincident mass mortality in the geoduck larvae was very likely a direct cause of the ciliate infestation. The evidence for this causal link is the observation of numerous ciliates coincident with the stress response and morality; the known link between ciliates and larval mortality in Pacific Northwest hatcheries ${ }^{31}$; and the increased abundance of the protozoan protein homologous to cruzipain at days 8 and 10 post-fertilization. In larvae reared in the same hatchery over the same period, but at lower $\mathrm{pH}$ (7.5 and 7.1), ciliates were not as prevalent as they were at $\mathrm{pH} 8.2$ (unpublished data) and a mass mortality was not observed. Since the larvae across all $\mathrm{pH}$ were reared in the same water and a mass mortality was only observed at $\mathrm{pH} 8.2$, it is unlikely that a bacterial or viral pathogen was the cause of larval mortality. A ciliate in the Family Orchitophyridae is a known primary pathogen of bivalve seed and larvae, infection by which leads to significant mortalities ${ }^{31}$. These ciliates were observed to enter oyster seed through the mantle cavity, where they proceeded to feed upon oyster tissue and fluids, destroying organs and leading to death ${ }^{31}$. Coincident with the ciliate infestation, the abundance of the protozoan protease homologous to cruzipain, used in the break-down of host tissues, was detected. Cruzipain is a protein that mediates infection in the parasites of the genus Trypanosoma ${ }^{32}$, but a homologous protein has been detected in parasites of divergent taxa as well ${ }^{33}$. A homologue of cruzipain is apparently also present in the ciliates observed in the hatchery to assist in larval tissue breakdown.

In a characterization of larval geoduck proteomes from day 4 through 10 post-fertilization, we were able to detect trends of normal physiological development as well as aberrations caused by a stress response to a ciliate infestation of the culture. Normal development was characterized by proteomic markers of tissue growth and upregulation of metabolism. Once the ciliates were detected, molecular signals of a stress response were observed, especially in the increased abundance of specific protein translation proteins, heat shock proteins and response to oxidative damage. These signals confirm that geoduck larvae have an inducible immune/stress response in the veliger stage, as has been observed in other bivalve larvae ${ }^{9}$. These universal markers can be used to detect and track the health of larval cultures in hatchery settings.

\section{Methods}

Larval rearing and ciliate outbreak. Broodstock maturation, spawning, and larval development (days 1-10 post-fertilization) occurred in a commercial hatchery in Hood Canal in Washington (USA) per normal operation conditions. Water temperature was maintained at approximately $14^{\circ} \mathrm{C}$, and larvae were reared in two $200 \mathrm{~L}$ conical tanks at a density of 70,000 fertilized eggs/L.

Larvae were maintained in seawater treated with $\mathrm{Na}_{2} \mathrm{CO}_{3}$ to maintain a $\mathrm{pH}$ of 8.2 and were sampled on days $4,6,8$, and 10 post-fertilization. However, on days 8 and 10 post-fertilization, a ciliate infestation was observed in both tanks. The infestation was characterized by an increase in number of ciliates observed between days 8 and 10 post-fertilization and increasing levels of larval mortality. After day 10 post-fertilization, the experiment had to be discontinued due to mortality. 
Given that ciliate infestation events have become more severe and frequent in bivalve commercial hatcheries in the US Pacific Northwest during recent years, and the cost associated with these outbreaks, we decided to take advantage of this event to characterize the immune/stress response of geoduck clam larvae to this opportunistic parasite ciliate infestation.

Larval sampling. On days $4,6,8$ and 10 post-fertilization, approximately 10,000 larvae were collected using a $20 \mu \mathrm{m}$ mesh screen, rinsed with $70 \%$ isopropyl alcohol, and stored at $-80^{\circ} \mathrm{C}$ for proteomics. Day 4 represented a bulk sample of larvae before they were divided between two tanks; for subsequent time points, larvae were sampled from $n=2$ tanks. For each sampling, larval size classes were determined using a series of mesh screens (from $90-200 \mu \mathrm{m}$ in $20-\mu \mathrm{m}$ intervals). The number of larvae retained in each screen was estimated by weight based on an established conversion developed in the hatchery (Supplementary Table S2).

Larvae were sampled on days $4,6,8$, and 10 post-fertilization for proteomic analysis as part of a parallel study at $\mathrm{pH} 7.1$ and $7.5^{23}$. Ciliate-associated mortality was not observed at either of these lower $\mathrm{pH}$ giving us a non-ciliate impacted proteome against which we could compare the proteome of larvae responding to ciliates.

Protein extraction and LC-MS/MS. Larval proteins were digested and peptides were desalted following ${ }^{34}$. Larval peptides were analyzed on an Orbitrap Fusion Lumos mass spectrometer (Thermo Scientific, Waltham, MA, USA) with a $4.5 \mathrm{~cm}, 100 \mu \mathrm{m}$ pre-column and a $26 \mathrm{~cm}, 75 \mu \mathrm{m}$ analytical column, both packed in-house with $3 \mu \mathrm{m} \mathrm{C18} \mathrm{Dr.} \mathrm{Maisch} \mathrm{(Germany)} \mathrm{beads.} \mathrm{The} \mathrm{analytical} \mathrm{column} \mathrm{was} \mathrm{housed} \mathrm{in} \mathrm{a} 50^{\circ} \mathrm{C}$ column heater for the duration of the analysis to improve chromatography. Over a 120 minute method, a 90 -minute acetonitrile gradient went from 5-30\%. In MS1 analysis in the Orbitrap, the resolution was $120 \mathrm{~K}$, scan range was $375-1575 \mathrm{~m} / z$, $\max$ injection time was $50 \mathrm{~ms}$, and AGC target was 700000. During the MS2 analysis in the IonTrap maximum injection time was $100 \mathrm{~ms}$, AGC target was 2000, and centroid data was collected. The mass spectrometry proteomics data have been deposited to the ProteomeXchange Consortium via the PRIDE ${ }^{35}$ partner repository with the dataset identifier PXD013667.

Proteomics analysis. All mass spectrometry raw files were searched against a deduced geoduck larvae proteome ${ }^{36}$ with the addition of common laboratory contaminants from bovine, human, and other (cRAPome). Redundancy of sequences in the larval proteome was reduced using CD-hit ${ }^{37}$. Details on reference proteome development are described elsewhere ${ }^{38}$. Comet ${ }^{39,40} 2016.01$ rev. 3 parameters included concatenated decoy search, mass tolerance of $20 \mathrm{ppm}, 2$ allowed missed trypsin cleavages, fragment bin tolerance of 1.0005 and offset of 0.4 . Peptide and Protein Prophet ${ }^{41,42}$ were run consecutively using xinteract with no probability cut-off to allow for FDR cut-off later in the pipeline. Resulting pep.xml files were analyzed in Abacus ${ }^{43}$ with a FDR cut-off of 0.01 (probability from combined prot.xml file of 0.9) (Supplementary Methods).

Nonmetric multidimensional scaling (NMDS) analysis was performed on all proteins that were inferred across technical mass spectrometry replicates with at least 2 unique peptide spectral matches. Protein abundance data (normalized spectral abundance factor) were $\log (\mathrm{x}+1)$ transformed and a Bray-Curtis dissimilarity matrix was calculated in R (https://www.r-project.org/). Eigenvectors were generated using envfit in the vegan package ${ }^{44}$. An eigenvector was considered significant if its axis loading value was $>0.9$ and its $p$-value was $<0.001$. ANOSIM was performed on normalized spectral abundance factor (NSAF) data standardized by row to assess significance of trends observed on the NMDS based on day.

Differentially abundant proteins between consecutive time points (e.g., day 6 vs day 8) were determined using Q $_{\text {spec }}{ }^{45}$. This analysis was not done for day 4 since there was just a single data point for that day. For each QSpec analysis, only proteins that had a non-zero sum of spectral counts across replicates were included and spectral counts were summed across technical replicates. Proteins were considered differentially abundant if they had a QSpec log-fold change of at least $|0.5|$ and a $\mathrm{z}$-statistic of at least $|2|$.

Based on homology of protein annotations, 11 differentially abundant proteins were considered to be involved in larval response to ciliates and one protein (cruzipain) was likely an indicator of ciliate proliferation. These proteins were grouped using a hierarchical clustering method based on NSAF values over time. NSAF values were averaged across conical replicates and mass spectrometry technical replicates within days and a Bray-Curtis dissimilarity matrix was calculated in $\mathrm{R}$ using the average clustering method. Cluster division was based on dendrogram topology (cut-off $=0.5$ ). Cluster abundance traces were visualized using ggplot $2^{46}$.

Enrichment analysis was performed using an in-house Gene Ontology (GO) term enrichment tool described in $^{47}$ via the project-specific portal https://meta.yeastrc.org/compgo_emma_pgenph8/pages/goAnalysisForm.jsp. The over-representation of GO categories (cellular component, biological process, and molecular function) was assessed in proteins that were differentially abundant between days or detected only on a particular day with a p-value cut-off of $1 \mathrm{E}-1$.

Proteins identified in the $\mathrm{pH} 8.2$ dataset were compared with proteins inferred from the low $\mathrm{pH}$ study ${ }^{23}$ to identify proteins that may be involved in the immune response to ciliates since no ciliate-induced mortality was observed at lower $\mathrm{pH}$. These proteins were subjected to enrichment analysis and cross-referenced with the differentially abundant proteins.

\section{Data availability}

The proteomic dataset generated during and/or analyzed during the current study is available in the ProteomeXchange Consortium repository via the PRIDE partner repository with the dataset identifier PXD013667.

Received: 10 October 2019; Accepted: 31 January 2020;

Published online: 08 April 2020 


\section{References}

1. Bassim, S., Genard, B., Gauthier-Clerc, S., Moraga, D. \& Tremblay, R. Ontogeny of bivalve immunity: assessing the potential of nextgeneration sequencing techniques. Reviews in Aquaculture 7, 197-217 (2015).

2. Tirapé, A., Bacque, C., Brizard, R., Vandenbulcke, R. \& Boulo, V. Expression of immune-related genes in the oyster Crassostrea gigas during ontogenesis. Developmental \& Comparative Immunology 31, 859-873 (2007).

3. Elston, R. A. Infectious diseases of the Pacific oyster, Crassostrea gigas. Annual Review of Fish Diseases 3, 259-276 (1993).

4. Paillard, C., Le Roux, F. \& Boreego, J. J. Bacterial disease in marine bivalves, a review of recent studies: Trends and evolution. Aquatic Living Resources 17, 477-498 (2004).

5. Balseiro, P., Moreira, R., Chamorro, R., Figueras, A. \& Novoa, B. Immune responses during the larval stages of Mytilus galloprovincialis: Metamorphosis alters immunocompetence, body shape and behavior. Fish \& Shellfish Immunology 35, 438-447 (2013).

6. Dyrynda, E. A., Pipe, R. K. \& Ratcliffe, N. A. Host defence mechanisms in marine invertebrate larvae. Fish \& Shellfish Immunology 5, 569-580 (1995).

7. Genard, B., Pernet, F., Lemarchand, K. \& Boudry, P. Physiological and biochemical changes associated with massive mortality events occurring in larvae of American oyster (Crassostrea virginica). Aquatic Living Resources 24, 247-260 (2011).

8. Genard, B. et al. Expression of candidate genes related to metabolism, immunity and cellular stress during massive mortality in the American oyster Crassostrea virginica larvae in relation to biochemical and physiological parameters. Gene 499, 70-75 (2012).

9. Genard, B. et al. Integrative Study of Physiological Changes Associated with Bacterial Infection in the Pacific Oyster Larvae. PLoS ONE 8, e64534, https://doi.org/10.1371/journal.pone.0064534 (2013).

10. Song, X. et al. The immunological capacity in the larvae of Pacific oyster Crassostrea gigas. Fish \& Shellfish Immunology 49, 461-469 (2016).

11. Toews, S., Beverley-Burton, M. \& Lawrimore, T. Helminth and protist parasites of zebra mussels, Dreissena polymorpha (Pallas, 1771), in the Great Lakes region of southwestern Ontario, with comments on associated bacteria. Canadian Journal of Zoology 71 , 1763-1766 (1993).

12. Hine, P. M. \& Thorne, T. A survey of some parasites and diseases of several species of bivalve mollusc in northern Western Australia. Diseases of Aquatic Organisms 40, 67-78 (2000).

13. Vázquez, N., Ituarte, C. \& Cremonte, F. A histopathological study of the geoduck clam Panopea abbreviata from San José Gulf, North Patagonia, Argentina. Journal of the Marine Biological Association of the United Kingdom 6, 1173-1181 (2015).

14. Shimeta, J., Cutajar, J., Watson, M. G. \& Vlamis, T. Influences of biofilm-associated ciliates on the settlement of marine invertebrate larvae. Marine Ecology Progress Series 449, 1-12 (2012).

15. Elston, R.A. Shellfish Diseases and Their Management in Commercial Recirculating Systems. Proceedings The Second International Conference on Recirculating Aquaculture pp. 147-149 (1998).

16. Coan, E.V., Scott, P.V., \& Bernard, F.R. Bivalve Seashells of Western North America: Marine Bivalve Mollusks from Arctic Alaska to Baja California. (Santa Barbara Museum of Natural History, 2000).

17. Vadopalas, B., Pietsch, T. W. \& Friedman, C. S. The Proper Name for the Geoduck Resurrection of Panopea generosa, from the Synonymy of Panopea abrupta (Bivalvia: Myoida: Hiatellidae). Malacologia 52, 169-173 (2010).

18. González-Peláez, S. S., Leyva-Valencia, I., Pérez-Valencia, S. \& Lluch-Cota, D. B. Distribution Limits of the Geoduck Clams Panopea generosa and P. globosa on the Pacific Coast of Mexico. Malacologia 56, 85-94 (2013).

19. Newell, R. I. Ecosystem influences of natural and cultivated populations of suspension-feeding bivalve mollusks: A review. Journal of Shellfish Research 23, 51-62 (2004).

20. Straus, K., Crosson, L. \& Vadopalas, B. Effects of Geoduck Aquaculture on the Environment: A Synthesis of Current Knowledge. (School of Aquatic and Fishery Sciences, University of Washington (2009).

21. Kent, M. L., Elston, R. A., Nerad, T. A. \& Sawyer, T. K. An Isonema-like flagellate (Protozoa: Mastigophora) infection in larval geoduck clams, Panope abrupta. Journal of Invertebrate Pathology 40, 221-229 (1987).

22. Dorfmeier, E. M., Vadopalas, B., Frelier, P. \& Friedman, C. S. Temporal and Spatial Variability of Native Geoduck (Panopea generosa) Endosymbionts in the Pacific Northwest. Journal of Shellfish Research 34, 81-90 (2015).

23. Timmins-Schiffman, E., Guzmán, J.M., Elliott, R., Vadopalas, B., \& Roberts, S.B. Dynamic response in the larval geoduck clam proteome to elevated pCO2. Ecology and Evolution https://doi.org/10.1002/ece3.5885 (2019).

24. Timmins-Schiffman, O’Donnell, M.J., Friedman, C.S., \& Roberts, S.B. Elevated pCO2 causes developmental delay in early larval Pacific oysters, Crassostrea gigas. Marine Biology 160, 1973-1982 (2013).

25. Roberts, R. J., Agius, C., Saliba, C., Bossier, P. \& Sung, Y. Y. Heat shock proteins (chaperones) in fish and shellfish and their potential role in relation to fish health: a review. Journal of Fish Diseases 33, 789-801 (2010).

26. Mailloux, R. J., McBride, S. L. \& Harper, M.-E. Unearthing the secrets of mitochondrial ROS and glutathione in bioenergetics. Trends in Biochemical Sciences 38, 592-602 (2013).

27. Bachère, E. et al. The new insights into the oyster antimicrobial defense: Cellular, molecular and genetic view. Fish \& Shellfish Immunology 46, 50-64 (2015).

28. Gestal, C. et al. Study of Diseases and the Immune System of Bivalves Using Molecular Biology and Genomics. Reviews in Fisheries Sciences sup1, 133-156 (2008).

29. Mohr, I. \& Sonenberg, N. Host Translation at the Nexus of Infection and Immunity. Cell Host \& Microbe 12, 470-483 (2012).

30. Zhang, K. \& Kaufman, R. J. From endoplasmic-reticulum stress to the inflammatory response. Nature 454, 455-462 (2008).

31. Elston, R. A., Cheney, D., Frelier, P. \& Lynn, D. Invasive orchitophryid ciliate infections in juvenile Pacific and Kumomoto oysters, Crassostrea gigas and Crassostrea sikamea. Aquaculture 174, 1-14 (1999).

32. Lima, L. et al. Repertoire, Genealogy and Genomic Organization of Cruzipain and Homologous Genes in Trypanosoma cruzi, T. cruzi-Like and Other Trypanosome Species. PLoS ONE 7, e38385, https://doi.org/10.1371/journalpone.0038385 (2012).

33. Yun, D.-H. et al. Structural and Immunological Characteristics of a 28-Kilodalton Cruzipain-Like Cysteine Protease of Paragonimus westermani Expressed in the Definitive Host Stage. Clinical and Diagnostic Laboratory Immunology 7, 932-939 (2000).

34. Timmins-Schiffman, E., Nunn, B. L., Goodlett, D. R. \& Roberts, S. B. Shotgun proteomics as a viable approach for biological discovery in the Pacific oyster. Conservation Physiology 1, cot009, https://doi.org/10.1093/conphys/cot009 (2013).

35. Perez-Riverol, Y. et al. The PRIDE database and related tools and resources in 2019: improving support for quantification data. Nucleic Acids Research 47, D442-D450 (2019).

36. Timmins-Schiffman, E. et al. Data Accompanying "Larval Geoduck (Panopea generosa) Proteomic Response to Ciliates". Figshare https://figshare.com/s/09075c03e0157c2fe6ec (2019).

37. Li, W., jaroszewski, L. \& Godzik, A. Clustering of highly homologous sequences to reduce the size of large protein databases. Bioinformatics 17, 282-283 (2001).

38. Roberts, S.G. paper-Pg-larvae-2019. GitHub https://github.com/sr320/paper-Pg-larvae-2019 (2019).

39. Eng, J. K., Jahan, T. A. \& Hoopmann, M. R. Comet: An open-source MS/MS sequence database search tool. Proteomics 13, 22-24 (2013).

40. Eng, J. K. et al. A Deeper Look into Comet - Implementation and Features. Journal of the American Society for Mass Spectrometry 26, 1865-1874 (2015).

41. Keller, A., Nesvizhskii, A. I., Kolker, E. \& Aebersold, R. Empirical Statistical Model to Estimate the Accuracy of Peptide Identifications Made by MS/MS and Database Search. Analytical Chemistry 74, 5383-5392 (2002). 
42. Nesvizhskii, A. I., Keller, A., Kolker, E. \& Aebersold, R. A Statistical Model for Identifying Proteins by Tandem Mass Spectrometry. Analytical Chemistry 75, 4646-4658 (2003).

43. Fermin, D., Basrur, V., Yocum, A. K. \& Nesvizhskii, A. I. Abacus: A computations tool for extracting and pre-processing spectral count data for label-free quantitative proteomic analysis. Proteomics 11, 1340-1345 (2011).

44. Oksanen, J. et al. vegan: Community Ecology Package. R package https://CRAN.R-project.org/package=vegan.

45. Choi, H., Fermin, D. \& Nesvizhskii, A. I. Significance Analysis of Spectral Count Data in Label-Free Shotgun Proteomics. Molecular \& Cellular. Proteomics 7, 2373-2385 (2008).

46. Wickham, H. ggplot2: Elegant Graphics for Data Analysis. Springer-Verlag New York (2009)

47. Timmins-Schiffman, E.B. et al. Integrating Discovery-driven Proteomics and Selected Reaction Monitoring to Develop a Noninvasive Assay for Geoduck Reproductive Maturation. Journal of Proteome Research 16, 3298-3309.

\section{Acknowledgements}

This work was funded in part by a grant from Washington Sea Grant, University of Washington, pursuant to National Oceanic and Atmospheric Administration Award No. NA14OAR4170078 Project R/SFA-8. The views expressed herein are those of the authors and do not necessarily reflect the views of NOAA or any of its subagencies. We would like to thank Jimmy Eng, Vagisha Sharma, and Michael Riffle for assistance with data analysis. Brook Nunn provided analytical guidance and Michael MacCoss provided space for completing this project. Mass spectrometry was completed at and supported in part by the University of Washington Proteomics Resource (UWPR 95794) with the assistance of Priska von Haller. Thank you to IJE for continuing inspiration.

\section{Author contributions}

E.T.S. was involved in data collection and was responsible for data analysis and interpretation and writing the manuscript. J.M.G. was responsible for sample prep for mass spectrometry, mass spectrometry data collection, and assisted with data interpretation and drafting of the manuscript. R.E.T. was responsible for carrying out the experiment in the shellfish hatchery and assisted in manuscript preparation. B.V. was responsible for experimental design and assisted in drafting of the manuscript. B.E. supervised the experiment at the hatchery and was involved in drafting of the manuscript. S.B.R was responsible for experimental design with B.V. and contributed to data interpretation and drafting of the manuscript.

\section{Competing interests}

The authors declare no competing interests.

\section{Additional information}

Supplementary information is available for this paper at https://doi.org/10.1038/s41598-020-63218-x.

Correspondence and requests for materials should be addressed to S.B.R.

Reprints and permissions information is available at www.nature.com/reprints.

Publisher's note Springer Nature remains neutral with regard to jurisdictional claims in published maps and institutional affiliations.

(c) (1) Open Access This article is licensed under a Creative Commons Attribution 4.0 International License, which permits use, sharing, adaptation, distribution and reproduction in any medium or format, as long as you give appropriate credit to the original author(s) and the source, provide a link to the Creative Commons license, and indicate if changes were made. The images or other third party material in this article are included in the article's Creative Commons license, unless indicated otherwise in a credit line to the material. If material is not included in the article's Creative Commons license and your intended use is not permitted by statutory regulation or exceeds the permitted use, you will need to obtain permission directly from the copyright holder. To view a copy of this license, visit http://creativecommons.org/licenses/by/4.0/.

(C) The Author(s) 2020 